\title{
The Importance of the Unsteady Kutta Condition when Modelling Gust-Aerofoil Interaction
}

\author{
Lorna J. Ayton ${ }^{\mathrm{a}}$, J. R. Gill ${ }^{\mathrm{b}}$, N. Peake ${ }^{\mathrm{a}}$ \\ ${ }^{a}$ Department of Applied Mathematics and Theoretical Physics, University of Cambridge, \\ Wilberforce Road, CB3 OWA, UK \\ ${ }^{b}$ Airbus Noise Technology Centre, University of Southampton, Burgess Road, \\ Southampton, SO16 $7 Q F$
}

\begin{abstract}
The Kutta condition is applied to aerofoils with sharp trailing edges to allow for viscous effects to be considered within a simplified system of equations that are inviscid. This paper discusses in detail the inclusion of an unsteady Kutta condition at a sharp trailing edge during gust-aerofoil interaction, and illustrates how the analytic solution for the far-field noise generated by this interaction changes if the unsteady Kutta condition is neglected, or more precisely, if the unsteady pressure is permitted to be singular at the trailing edge. The analytic solution, both with and without the unsteady Kutta condition, is compared with numerical results that have no imposed unsteady Kutta condition. Importantly the results agree well only when the unsteady Kutta condition is neglected in the analytic solution. This paper highlights where the far-field acoustics are most affected by neglecting the unsteady Kutta condition for a variety of singularities that can occur in the unsteady pressure at the trailing edge, and shows that results permitting different behaviour in the unsteady surface pressure at the trailing edge could give significantly different far-field noise predictions, even though the surface pressure elsewhere along the aerofoil surface agrees with benchmark solutions.
\end{abstract}

Keywords: leading-edge noise, Kutta condition

Email address: L.J.Ayton@damtp.cam.ac.uk (Lorna J. Ayton) 


\section{Introduction}

The unsteady Kutta condition is a well-known boundary condition applied at the trailing edge of aerofoils when investigating acoustic flow interactions (see Crighton [9] for a review). In the context of this paper we take it to enforce that the pressure jump across the trailing edge of the aerofoil is zero, and singular velocities and pressures are not permitted at the trailing edge. It allows potential-flow theory to be used in preference to the full Navier-Stokes equations for high Reynolds number interactions, which is particularly beneficial for obtaining analytical solutions to unsteady interaction problems. For the case of the full Navier-Stokes equations, the Kutta condition is thought to be valid in laminar boundary layers when unsteady (non-dimensionalised) frequencies are less than $O\left(R e^{1 / 4}\right)[9]$ where $R e$ is the Reynolds number based on the chord length. In this paper we are concerned with potential-flow interactions, in particular the noise generated by gust-aerofoil interaction in steady flow (uniform far upstream), which has important applications to rotor-stator interaction noise within turbo-machinery [27].

Analytically the unsteady Kutta condition is simple to impose, along with the zero-normal velocity on the solid aerofoil surface. For flat plates in uniform steady flow at zero angle of attack with an unsteady incident gust, Howe [19] and Amiet [3] discuss the analytic gust response function and the effect of the unsteady Kutta condition at the trailing edge. In particular, Amiet [3] quotes "for inviscid flow the Kutta condition is satisfied automatically as the gust passes the trailing edge". It is however less clear exactly what effect the Kutta condition has on aerofoils with non-zero thickness or camber, and this is a key problem in order to accurately predict gust-aerofoil interactions in realistic situations. Also less clear is the most appropriate way to impose the Kutta condition numerically and if it is required in real-geometry situations; a variety of different techniques exist to impose the condition $[6,8,10,21]$, and we draw particular attention to Bose [8, Fig. 2] which compares two different numerical Kutta conditions, which yield different surface pressure results at the trailing edge. A number of numerical methods do not impose the condition $[1,15,17,22,30]$, and instead only a zero-normal velocity condition is imposed.

The unsteady Kutta condition acts to control the unsteady velocities and pressures at the trailing edge, therefore in this paper we pay particular attention to the surface pressure at the trailing edge. Various numerical

results that do not impose an unsteady Kutta condition show singular or 
"spiked" trailing-edge pressures even though the pressure along the remaining aerofoil chord agrees with benchmark solutions [1, 17, 22, 30]. Only a few of these papers go on to compare far-field noise predictions with previous results, $[30,17]$, and we see in these far-field results discrepancy between different codes that do or do not have the unsteady Kutta condition imposed. Whilst it has been reported in Sandberg \& Sandham [28] and Sandberg et al. [29] that the effects of the unsteady Kutta condition on the far-field noise can be neglected, we highlight that this is only shown for flat plates, and not for aerofoils with realistic thickness or camber (such as the cases in [30, 17]), and indeed Amiet [3] predicts that the unsteady Kutta condition is not a necessity for flat plates as it is self-imposed by the solution. It has been shown analytically [32] and numerically [13] that introducing non-zero thickness has a significant effect on sound generation for aerofoils of finite chord length, therefore one should be cautious about classifying zero-thickness and nonzero-thickness aerofoils in the same way.

We therefore investigate the effect of the unsteady Kutta condition on the sound generated by gust-aerofoil interaction, for aerofoils with non-zero thickness, analytically. We use this to determine whether singularities or spikes appearing in numerical surface pressure results (due to the lack of an unsteady Kutta condition) can affect the far-field acoustic results. We do so by considering one particular numerical solution in detail, first presented in Gill et al. [13], in which no unsteady Kutta condition is enforced and therefore there is a singularity in the unsteady surface pressure at the trailing edge.

We discuss the analytical solution for gust-aerofoil interaction in Section 2, and concentrate on the Wiener-Hopf solution for the trailing-edge interaction in Section 3, highlighting where the unsteady Kutta condition is imposed, and how the solution changes if we neglect it. We shall find that some levels of singularity in the pressure jump across the trailing edge do not alter the far-field acoustics, while others can have significant effects. Section 4 reviews the numerical method from Gill et al. [13], and Section 5 contains results illustrating the effect of neglecting the unsteady Kutta condition on the far-field acoustic pressure, along with a comparison of the analytical solution with the numerical results in which the unsteady Kutta condition is not imposed. Note the steady Kutta condition is imposed in the numerical result. Section 6 contains concluding remarks. 


\section{Analytical Solution for Gust-Aerofoil Interaction}

Gust-aerofoil interaction noise, and more generally leading-edge noise, is a popular problem to study numerically $[17,13]$, experimentally $[12,11]$ and analytically $[2,25,32]$. The analytical solution for gust-aerofoil interaction in uniform flow from Myers \& Kerschen [25] considers only zero-thickness aerofoils, whilst the solution from Tsai [32] considers only symmetric aerofoils with non-zero thickness. In this section we present a brief overview of the solution for an aerofoil with small but non-zero thickness, camber and angle of attack (however later we simplify for a zero-camber case to illustrate the effects of the unsteady Kutta condition on non-zero thickness aerofoils). These small parameters scale with $\epsilon \ll 1$, where lengths are non-dimensionalised with respect to the aerofoil semi-chord length, $b^{*}$. Specifically we focus on high-frequency incident gusts, with reduced frequency $2 \pi b^{*}\left(\lambda^{*}\right)^{-1}=k \gg 1$ (where $\lambda^{*}$ is the wavelength of the gust) and impose $\epsilon k=O(1)$, in line with Myers \& Kerschen [25] and Tsai [32]. Further details of the model can be found in Ayton \& Peake [5] and Ayton [4].

We study the interaction using rapid distortion theory [14]. The governing equation for the modified unsteady velocity potential, $h$, generated when a gust, of the form $\left(A_{t}, A_{n}, A_{3}\right) \mathrm{e}^{\mathrm{i} k\left(k_{t} \phi+k_{n} \psi+k_{3} z-k_{t} t\right)}$ far upstream, interacts with an aerofoil in uniform flow with Mach number $M_{\infty}$ is given by

$$
\begin{gathered}
\frac{\partial^{2} h}{\partial \phi^{2}}+\frac{\partial^{2} h}{\partial \psi^{2}}+k^{2} w^{2}\left(1-2 \beta_{\infty}^{2} \epsilon q\right) h+\frac{(\gamma+1) M_{\infty}^{4} \epsilon q}{\beta_{\infty}^{2}}\left(\frac{\partial^{2} h}{\partial \psi^{2}}+2 i k \delta \frac{\partial h}{\partial \phi}+k^{2}\left(w^{2}+\delta^{2}\right) h\right) \\
-\frac{(\gamma+1) M_{\infty}^{4} \epsilon}{\beta_{\infty}^{2}} \frac{\partial q}{\partial \phi}\left(\frac{\partial h}{\partial \phi}-\mathrm{i} k \delta h\right)=k \epsilon S(\phi, \psi) \mathrm{e}^{\mathrm{i} k \Omega}, \quad \text { (1a) }
\end{gathered}
$$

where

$$
\delta=k_{t} / \beta_{\infty}^{2}, \quad w^{2}=\left(M_{\infty} \delta\right)^{2}-\left(k_{3} / \beta_{\infty}\right)^{2}, \quad \Omega=\delta \phi+k_{n} \psi+\epsilon g(\phi, \psi),
$$

and

$S(\phi, \psi)=\frac{2}{\beta_{\infty}^{2}}\left(\mathrm{i}\left(A_{t}-A_{n} k_{n} \beta_{\infty}^{3}\right) q+\mathrm{i}\left(\beta_{\infty}^{2} k_{n} A_{t}+A_{n} \beta_{\infty}\right) \mu+\frac{A_{t} M_{\infty}^{2}}{k} \frac{\partial q}{\partial \phi}+\frac{A_{n} M_{\infty}^{2} \beta_{\infty}}{k} \frac{\partial q}{\partial \psi}\right)$.

The function, $g(\phi, \psi)$, is Lighthill's drift function,

$$
g(\phi, \psi)=-2 \int_{-\infty}^{\phi} q(\eta, \psi) d \eta
$$


and the boundary condition of zero normal velocity on the solid surface is

$$
\frac{\partial h}{\partial \psi}+\left.M_{\infty}^{2} \epsilon \frac{\partial q}{\partial \psi} h\right|_{\psi=0}=\left.\left(-\frac{A_{n}}{\beta_{\infty}}+2 \epsilon \mu A_{t}+\frac{A_{n} M_{\infty}^{2} \epsilon q}{\beta_{\infty}}\right) \mathrm{e}^{\mathrm{i} k \Omega}\right|_{\psi=0} .
$$

The functions $q(\phi, \psi)$ and $\mu(\phi, \psi)$ describe the perturbation to the steady flow due to the presence of the aerofoil and are defined by Thwaites [31] as

$$
(q-i \mu)(\zeta)=\frac{1}{\pi \beta_{\infty}} \int_{0}^{2} \frac{y^{(t) \prime}(x)}{\zeta-x} d x+\frac{1}{\pi \beta_{\infty}} \sqrt{\frac{2-\zeta}{\zeta}} \int_{0}^{2} \frac{y^{(c) \prime}(x)}{\zeta-x} \sqrt{\frac{x}{2-x}} d x
$$

where $\zeta=\phi+\mathrm{i} \psi$, and the aerofoil boundary is given by $\epsilon y(x)= \pm \epsilon y^{t}(x)+$ $\epsilon y^{c}(x)$, where $y^{t, c}$ corresponds to the thickness and camber distributions respectively.

The coordinates, $\phi$ and $\psi$ (non-dimensionalised by $b^{*} U_{\infty}^{*}$, where $U_{\infty}^{*}$ is the steady flow speed), are potential-streamline coordinates, which have the effect of mapping the aerofoil in Cartesian coordinates to a flat plate in $(\phi, \psi)$ coordinates. The spanwise coordinate is $z$, and we suppose the aerofoil extends infinitely in this direction. $\epsilon q$ is the perturbation to the steady uniform flow caused by the presence of the aerofoil, $\epsilon \mu$ denotes the perturbation angle the total steady flow makes with the physical $x$ axis, and $\beta_{\infty}=\sqrt{1-M_{\infty}^{2}}$ is the Prandtl-Glauert transformation factor. The forcing, $S$, arises from the evolution of the gust in the steady flow.

The boundary condition, (2), is applied on the solid surface, $\psi=0,0<$ $\phi<2+\phi_{e}$, where $2+\phi_{e}$ denotes the location of the trailing edge of the aerofoil under the transformation from physical space to $(\phi, \psi)$-space. Since this paper discusses only symmetric aerofoils at zero angle of attack, from now on we assume $y^{c}(x)=0$.

For $\zeta=O(1)$, i.e. away from the leading edge of the aerofoil, $q, \mu=O(1)$. Close to the leading edge, $\zeta=O\left(k^{-1}\right)$, there is a new scaling, $q, \mu=O\left(k^{-1 / 2}\right)$, which gives rise to the leading-edge inner region that assesses the distortion of the gust near the leading edge. This is typical of analytic models for gustaerofoil interaction using rapid distortion theory [25, 32], and hence to solve this problem we use the method of matched asymptotic expansions. All of the asymptotic regions required for this problem are illustrated in Figure 1. Importantly, within the inner regions at the leading and trailing edges, the solid aerofoil appears to be semi-infinite in the downstream and upstream directions respectively, therefore the problem is solved in these regions using the Wiener-Hopf method [26]. 


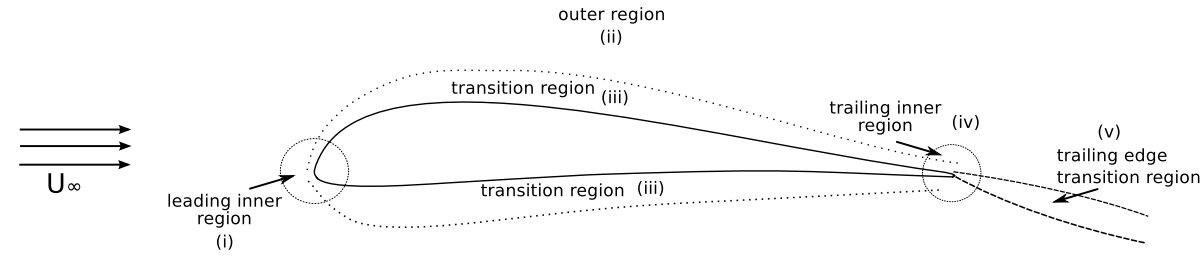

(a) In $(x, y)$ space.

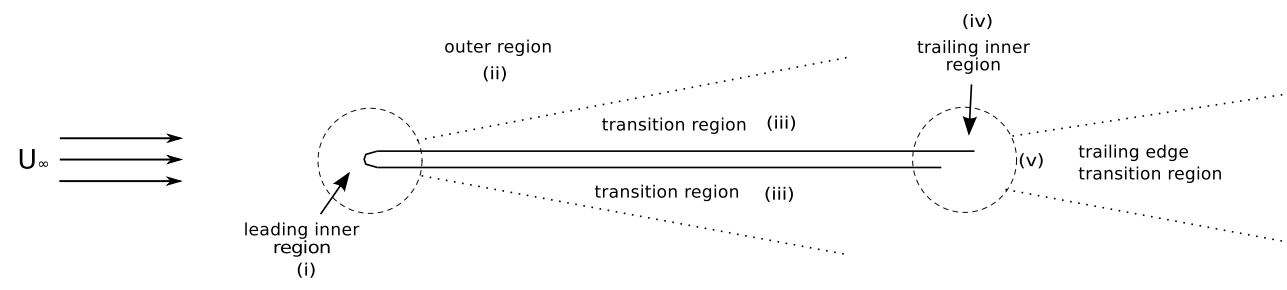

(b) In $(\phi, \psi)$ space.

Figure 1: Asymptotic regions around the aerofoil; leading- and trailing-edge inner regions, (i) and (iv), scale as $O\left(k^{-1}\right)$, and the width of the transition regions, (iii) scale as $O\left(k^{-1 / 2}\right)$. The outer region (ii) is $O(1)$.

The leading-edge inner solution, in region (i), is obtained by converting the governing equation to inner coordinates $(\Phi, \Psi)=k(\phi, \psi)$. The boundary condition is imposed on $\Psi=0, \Phi>0$ and a condition of continuous upstream pressure is imposed on $\Psi=0, \Phi<0$, where we use the modified unsteady pressure, as defined by Myers \& Kerschen [24, eq. (2.7)], given by

$$
p=-\left(\frac{\partial h}{\partial \phi}-\mathrm{i} k \delta h\right) \mathrm{e}^{-\mathrm{i} k \delta M_{\infty}^{2} \phi} .
$$

The leading-edge transition region, (iii), ensures the normal velocity is zero along the entire length of the aerofoil, and the solution here is found to exponentially decay in the far field, $r \gg 1$. The trailing-edge inner solution, in region (iv), is obtained in trailing-edge inner coordinates, $\left(\Phi_{t}, \Psi_{t}\right)=$ $k\left(\phi-2-\phi_{e}, \psi\right)$. The boundary condition is imposed along $\Psi_{t}=0, \Phi_{t}<0$. We assume the wake is a vortex sheet extending downstream from the trailing edge, so we require continuity of pressure across the wake; the solution emanating from the leading edge creates a discontinuity of pressure across the wake, which we must correct at the trailing edge. In the analytical model, we impose $[p]_{\Psi_{t}=0_{-}}^{\Psi_{t}=0_{+}}=-\left[p_{l}\right]_{\Psi_{t}=0_{-}}^{\Psi_{t}=0_{+}}$for $\Phi_{t}>0$, where $p_{l}$ is the pressure associated with the leading-edge solution. The unsteady Kutta condition arises 
at the trailing edge when we assume the velocity and pressure jump across the trailing edge are finite. The trailing-edge transition region, (v), ensures continuity of pressure and velocity across the wake.

The overall solution for the far-field noise generated by gust-aerofoil interaction in the outer region, (ii), consists of a contribution propagating from the leading edge due to the interaction of the solid surface and the gust, and a contribution from the trailing edge due to the rescattering of the leadingedge acoustics by the rigid edge. Since the trailing-edge field is key to the far-field acoustics it is incredibly important to ensure that the trailing edge is correctly modelled. This is investigated in the following section.

\section{The Wiener-Hopf Solution at the Trailing Edge}

The problem at the trailing edge is given by

$$
\frac{\partial^{2} H_{t}}{\partial \Phi_{t}^{2}}+\frac{\partial^{2} H_{t}}{\partial \Psi_{t}^{2}}+w^{2} H_{t}=0
$$

where $H_{t}$ is the trailing-edge inner unsteady velocity potential that we wish to find. The pressure jump across the trailing edge generated by the leadingedge solution can be written as

$$
-\left[p_{l}\right]_{-}^{+}=\left.p_{l}\right|_{\psi=0-}-\left.p_{l}\right|_{\psi=0+}=\Delta p\left(\phi_{t}\right) \mathrm{e}^{\mathrm{i} k\left(w-\delta M_{\infty}^{2}\right) \phi_{t}}, \quad \phi_{t}>0 .
$$

We enforce continuity of displacement across the wake

$$
\left.\frac{\partial H_{t}}{\partial \Psi_{t}}\right|_{\substack{\Phi_{t}>0 \\ \Psi_{t}=0+}}=\left.\frac{\partial H_{t}}{\partial \Psi_{t}}\right|_{\substack{\Phi_{t}>0 \\ \Psi_{t}=0-}}
$$

and the continuity of pressure condition, (6), in trailing-edge inner coordinates is

$$
\left[\frac{\partial H}{\partial \Phi_{t}}-i \delta H_{t}\right]_{\substack{\Phi_{t}>0 \\ \Psi_{t}=0-}}^{\substack{\Phi_{t}>0 \\ \Phi_{t}=0+}}=\lim _{k \rightarrow \infty} \Delta p\left(\Phi_{t} / k\right) \mathrm{e}^{\mathrm{i} w \Phi_{t}}
$$

for $\Phi_{t}>0$. The zero normal velocity condition on the aerofoil surface is satisfied by a hydrodynamic solution (found by Myers [23]), which does not affect the pressure condition nor radiate sound to the far field, therefore the acoustic solution at the trailing edge must satisfy

$$
\frac{\partial H_{t}}{\partial \Psi_{t}} \underbrace{}_{\substack{\Phi_{t}<0 \\ \Psi_{t}=0}}=0
$$


We also require the solution to be an outgoing acoustic wave thus impose a radiation condition.

We use the Wiener-Hopf method to solve the Helmholtz equation, (5), subject to (7), (8), and (9). We define the Fourier transform as

$$
\tilde{H}\left(\lambda, \Psi_{t}\right)=\int_{-\infty}^{\infty} H\left(\Phi_{t}, \Psi_{t}\right) \mathrm{e}^{\mathrm{i} \lambda \Phi_{t}} d \Phi_{t}
$$

therefore the Helmholtz equation yields the solution

$$
\tilde{H}\left(\lambda, \Psi_{t}\right)=\operatorname{sgn}\left(\Psi_{t}\right) A(\lambda) \mathrm{e}^{-\sqrt{\lambda^{2}-w^{2}}\left|\Psi_{t}\right|},
$$

where $A(\lambda)$ is an unknown function. Applying (9), we find

$$
-\sqrt{\lambda^{2}-w^{2}} A(\lambda)=F_{+}(\lambda)
$$

where

$$
F_{+}(\lambda)=\left.\int_{0}^{\infty} \frac{\partial H}{\partial \Psi_{t}}\right|_{\Phi_{t}>0} \mathrm{e}^{\mathrm{i} \lambda \Phi_{t}} d \Phi_{t}
$$

is unknown. We know that $F_{+}$is analytic in the upper half $\lambda$-plane since this is a positive half-range transform. Any such function that is analytic in the upper half plane is denoted by a subscript + whilst functions analytic in the lower half plane are denoted by a subscript - .

Using (8), we see that

$$
-2 \mathrm{i} A(\lambda)(\lambda+\delta)=\int_{-\infty}^{\infty} \Delta p\left(\Phi_{t}\right) \mathrm{e}^{\mathrm{i}(\lambda+w) \Phi_{t}} d \Phi_{t}=P_{+}(\lambda)+P_{-}(\lambda),
$$

where $P_{+}(\lambda)$ is known, and $P_{-}(\lambda)$ is unknown.

In $(12)$, we can divide through by $[\sqrt{\lambda+w}]_{+}$(we take the positive root in the definition of $w$ in (1b) and allow $w$ to have a small positive imaginary part), therefore the resulting left hand side must be analytic in the upper half plane, i.e. $\sqrt{\lambda-w} A(\lambda)=[\sqrt{\lambda-w} A(\lambda)]_{+}$.

Multiplying (14) by $\sqrt{\lambda-w}$ we obtain

$$
-2 \mathrm{i}(\lambda+\delta) A(\lambda) \sqrt{\lambda-w}=P_{+}(\lambda) \sqrt{\lambda-w}+\left[P_{-}(\lambda) \sqrt{\lambda-w}\right]_{-},
$$

where the left hand side is analytic in the upper half plane. On the right hand side of (15), we need to additively split $G(\lambda) \equiv P_{+}(\lambda) \sqrt{\lambda-w}$ into a plus term and a minus term, $G(\lambda)=G_{+}(\lambda)+G_{-}(\lambda)$. Therefore

$$
[-2 \mathrm{i}(\lambda+\delta) A(\lambda) \sqrt{\lambda-w}]_{+}-G_{+}(\lambda)=G_{-}(\lambda)+\left[P_{-}(\lambda) \sqrt{\lambda-w}\right]_{-} .
$$


The right hand side is analytic in the upper half plane, whilst the left hand side is analytic in the lower half plane. Therefore both sides must be equal to an entire function, $E(\lambda)$, which by Liouville's theorem is a polynomial in $\lambda$. To determine the coefficients of the polynomial, we must consider the large- $\lambda$ behaviour of (16). The continuity of displacement, (7), imposes $\frac{\partial H}{\partial \Psi_{t}} \rightarrow 0$ as $\Phi_{t} \rightarrow 0$, therefore $F_{+}(\lambda)=O\left(\lambda^{-1}\right)$ is the least singular behaviour of $F_{+}$for large $\lambda$, hence $A(\lambda)=O\left(\lambda^{-2}\right)$. The unsteady Kutta condition now arises as we find the behaviour of $P_{ \pm}(\lambda)$. Importantly, we cannot formally solve the Wiener-Hopf equation, (16), without additional information about the unknown function $P_{-}(\lambda)$.

\subsection{Imposing the Unsteady Kutta Condition}

Across the wake we have already imposed continuity conditions for the velocity and pressure via (7) and (8), however we have made no assumption about the behaviour of the pressure as it approaches the trailing edge along the aerofoil surface (as $\Phi_{t} \rightarrow 0_{-}$). We use the unsteady Kutta condition to consider the behaviour of $P_{ \pm}$for large $\lambda$ as required in the Wiener-Hopf method to determine the entire function, $E(\lambda)$, discussed at the end of the previous subsection. If we suppose the pressure jump, $\Delta p$, is finite at the trailing edge as $\Phi_{t} \rightarrow 0_{+}$(the wake side), then $P_{+} \sim(\lambda+w)^{-1}$ for large $\lambda$. In the analytical solution, $\Delta p$ is a constant for $\Phi_{t}>0$ and this is indeed the case. We impose the unsteady Kutta condition to tell us that the pressure jump is non-singular as $\Phi_{t} \rightarrow 0_{-}$across the trailing edge (the aerofoil side), hence we obtain a large $\lambda$ scaling of our unknown function, $P_{-}(\lambda) \sim O\left(\lambda^{-1}\right)$. Therefore the entire function, $E(\lambda) \rightarrow 0$ for large $\lambda$, and hence by Liouville's theorem the only possibility is $E(\lambda)=0$. Hence

$$
A(\lambda)=\frac{\mathrm{i} G_{+}(\lambda)}{2 \sqrt{\lambda-w}(\lambda+\delta)},
$$

and we obtain $H_{t}$ by inverting the Fourier transform. The far-field noise generated by the interaction at the trailing-edge is obtained by taking the outer limit of this inner solution and matching to the outer solution (region (ii) in Figure 1).

\subsection{Neglecting the Unsteady Kutta Condition}

If we do not impose the unsteady Kutta condition on the aerofoil side of the trailing edge, we cannot determine a scaling for the unknown $P_{-}(\lambda)$ 
when $\lambda \rightarrow \infty$, and therefore the entire function may be non-zero. If the pressure jump is singular at the trailing edge, $\Delta p=O\left(\Phi_{t}^{-n}\right)$ as $\Phi_{t} \rightarrow 0_{-}$ for some $0<n<1$, then $P_{-}(\lambda)=O\left(\lambda^{n-1}\right)$ for large $\lambda$ (using the method of stationary phase [7]). Therefore $E(\lambda)=O\left(\lambda^{[n-1 / 2]}\right)$, where [.] denotes the integer part. If the singularity has $n<1 / 2$ we would not expect to see an effect on the far-field acoustics; this could explain the results seen in Sandberg \& Sandham [28] which shows a flat plate without the unsteady Kutta condition generates very similar far-field acoustics to a flat plate with the imposed unsteady Kutta condition, despite a numerical surface pressure singularity present at the trailing edge when the unsteady Kutta condition is not imposed. For singularities with $n \geq 1, P_{-}(\lambda)=O\left(\lambda^{n-1} \log \lambda\right)$, therefore by the extended Liouville's theorem, $E(\lambda)$ is a polynomial of degree no more than $m$ where $m$ is such that $\lambda^{n-1 / 2} \log \lambda<c \lambda^{m}$ for large $\lambda$ and some constant c. Therefore we choose $m$ to be the integer strictly greater than $n-1 / 2$.

In the analytical solution we know $P_{+}(\lambda)$, so to retain consistency with the analytical solution we still suppose $P_{+} \sim(\lambda+w)^{-1}$ for large $\lambda$, but allow $P_{-}(\lambda)$ to scale differently since this is an unknown in the formal Wiener-Hopf equation. By allowing for a singularity in the surface pressure jump across the aerofoil trailing edge this modifies the solution for $A$;

$$
A(\lambda)=\frac{\mathrm{i} G_{+}(\lambda)}{2 \sqrt{\lambda-w}(\lambda+\delta)}+\frac{\mathrm{i} E(\lambda)}{2 \sqrt{\lambda-w}(\lambda+\delta)},
$$

which then affects the far-field noise generated at the trailing-edge.

We now give an example to illustrate this extra contribution. We suppose $1 / 2 \leq n<1$, so the entire function is a constant. Consider

$$
\Delta p \sim D\left(-\Phi_{t}\right)^{-n}
$$

as $\Phi_{t} \rightarrow 0_{-}$, therefore

$$
P_{-}(\lambda) \sim \frac{D \Gamma(1-n)}{(\mathrm{i}(\lambda+w))^{1-n}},
$$

hence the extra term added to $A(\lambda)$ is

$$
\frac{\mathrm{e}^{\pi \mathrm{i} n / 2} D \Gamma(1-n)}{2 \sqrt{(\lambda-w)}(\lambda+\delta)}
$$

which overall adds an additional term to the far-field noise emanating from the trailing edge (obtained by applying the method of steepest descents to 
the inverse Fourier transform of $\tilde{H}\left(\lambda, \Psi_{t}\right)$ and taking $\left.\left|\Phi_{t}\right|,\left|\Psi_{t}\right| \rightarrow \infty\right)$. This additional far-field noise is

$$
\frac{\mathrm{e}^{\mathrm{i} k w \sigma(r, \theta)}}{\sqrt{k r}} \frac{D|\sin (\theta / 2)| \Gamma(1-n) \mathrm{e}^{\pi \mathrm{i} n / 2}}{2 \sqrt{\pi}(\delta-w \cos \theta)},
$$

where $(r, \theta)$ are (outer) polar coordinates centred on the leading-edge of the aerofoil, and $\sigma$ is the far-field phase function for the trailing-edge acoustic solution.

We conclude from this analysis that not imposing the unsteady Kutta condition affects the trailing-edge field by producing a new contribution, (22). Physically this field ought not to be present, as the trailing-edge field should be produced only by the rescattering of the leading-edge field, which is given by (17). This additional contribution can be thought of as the scattering of an unphysical pressure field (the singularity) by the trailing edge. Since it is a scattered trailing-edge field, we see the $\sin (\theta / 2)$ directivity in (22), familiar to trailing-edge scattered fields [18].

The extra contribution to the far-field noise, (22), is dependent on $D$ and $n$ which represent the spike in the pressure at the trailing edge of the aerofoil. The only way to determine $D$ and $n$ is to compare the proposed singularity, (19) with the numerical pressure at the trailing edge. To illustrate the effects of a singularity verses the solution with imposed unsteady Kutta condition, we find suitable constants $D$ and $n$ by considering the numerical solution, first presented in Gill et al. [13], which calculates the surface pressure and far-field noise for gust-aerofoil interactions and does not impose an unsteady Kutta condition. This method is briefly outlined in the following section.

\section{Numerical Method}

The numerical method uses a high-order computational aeroacoustics (CAA) code to solve the linearized Euler equations (LEEs) in the timedomain. This solver and methodology has been previously used to predict turbulence-aerofoil interaction noise for a variety of aerofoil geometries and flow speeds [13]. The finite difference solver uses sixth-order spatial [16] and fourth-order temporal schemes [20] to solve the LEEs. Vortical waves are imposed at the inflow boundary of the simulation, where they are superimposed onto a steady and inviscid mean flow solution that convects the disturbances towards the aerofoil. Non-reflective buffer zone conditions are used at the domain edges to prevent spurious reflection at domain boundaries 


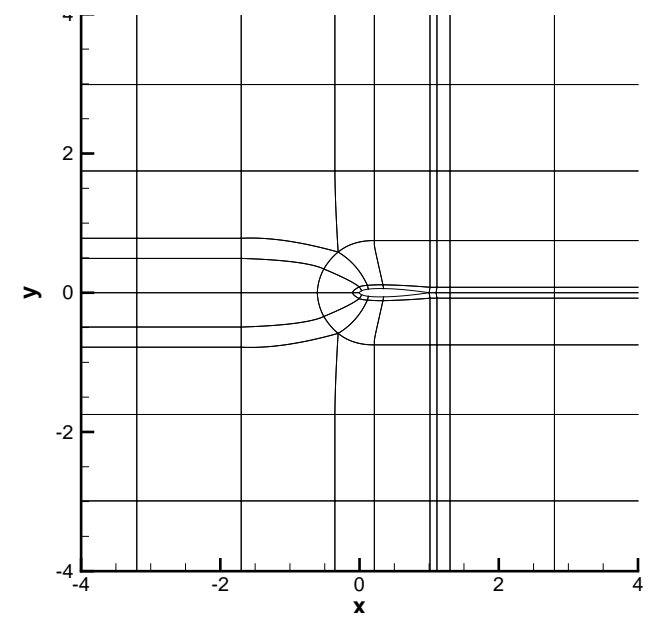

(a) Grid regions.

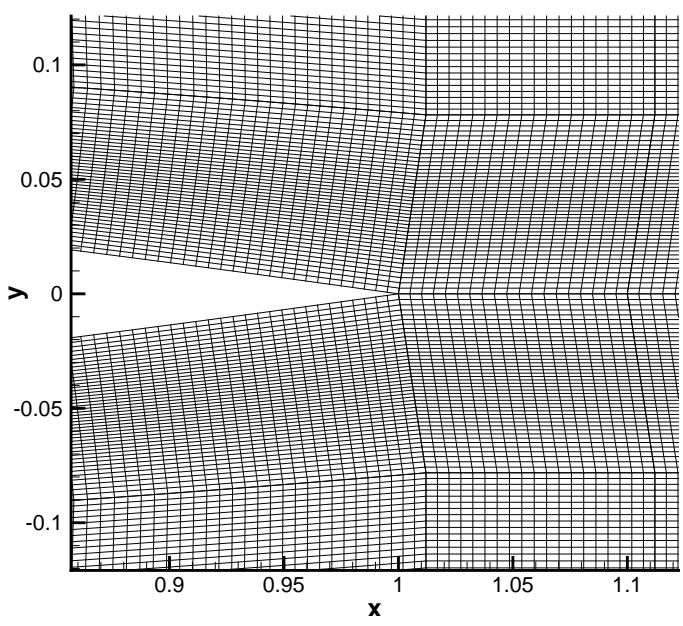

(b) Grid around trailing edge.

Figure 2: Grid used in the numerical code.

from interfering with the simulation results, and the domain extends to eight chord-lengths from the aerofoil in all directions. Far-field noise predictions are made using a Ffowcs-Williams and Hawkings (FW-H) solver, with the FW-H collection surface placed on the surface of the aerofoil. A grid resolution of at least 12 points-per-wavelength was used, and Figure 2 illustrates the grid used for case b) presented in the following section. The steady Kutta condition is imposed however the unsteady Kutta condition is not.

\section{Results}

In this section we consider the impact of neglecting the unsteady Kutta condition on the total far-field noise generated by gust-aerofoil interaction.

\subsection{Singularity in the Pressure}

Since the constant in (22) arises from a singularity in the pressure jump across the aerofoil as we approach the trailing edge, for each case we wish to consider we must choose the constant differently. The three cases we consider are a) NACA 0002 with $k=2 \pi$, b) NACA 0012 with $k=2 \pi$, c) NACA 0012 with $k=6 \pi$. In all cases $M_{\infty}=0.6$. Figure 3 illustrates the singularities that are present in the numerically determined pressure as $\Phi_{t} \rightarrow 0_{-}$, and the 
chosen best fitting functions of the form $D\left(-\Phi_{t}\right)^{-1 / 2}$. The choices of $D$ for cases a, b, and c are

$$
D_{a}=0, \quad D_{b}=0.3, \quad D_{c}=1.0 .
$$

We have fixed $n$ for each case for two reasons. First, it is easiest to investigate the case when $1 / 2 \leq n<1$ since the extra contribution then depends only on one constant. Second, it seems sensible that the type of singularity arising at the trailing edge in the numerical results is independent of thickness and frequency. $n=1 / 2$ is then chosen as a best fit to our cases of interest. With this choice, the $D_{i}$ scale as $O(\epsilon \sqrt{k})$, where $\epsilon$ is the aerofoil thickness parameter, indicating that the effects of the singularity may increase with thickness and frequency. As flow reaches the sharp trailing edge in the simulations, it is sharply turned from following along the aerofoil surface, to travelling in a direction (usually) aligned with the blade chord. For a flat plate, these two directions are the same. However, the thicker the aerofoil becomes, the greater amount of turning occurs. This sudden change in the velocity at the trailing edge is what we propose causes the spike in pressure jump, and thus the spike will increase with thickness.

The numerical result only calculates the real values of pressure, therefore as well as determining the value of $D$ we are free to choose a phase. We select this so that the overall (constant) phase for the extra term is $\mathrm{e}^{-\pi \mathrm{i} / 4}$, since there is a phase shift between the asymptotic first-order leading- and trailingedge acoustic sources of $\mathrm{e}^{-\pi \mathrm{i} / 4}$ (the remainder of the phase shift between the leading- and trailing-edge sources arises due to their differing spatial positions which will contain the phase term $\sigma(r, \theta)$ seen in $(22))$.

We note from Figure 3 that case a) does not have a singularity in the numerically calculated pressure jump at the trailing edge. Therefore, we expect the numerical result and the analytical solution (with imposed unsteady Kutta condition) to have good agreement. This is indeed seen in Figure 4a. We could have anticipated that the numerical solution satisfies the unsteady Kutta condition for the flat-plate case despite not specifically enforcing it since Amiet [2] predicts that symmetric flat-plate cases automatically satisfy the unsteady Kutta condition.

Figure 4 illustrates the effects of including the additional term in the analytical solution, and compares both analytical solutions with the numerical results for the far-field sound pressure level. In case a), where there was no spike in the numerical surface pressure at the trailing edge, we have good 


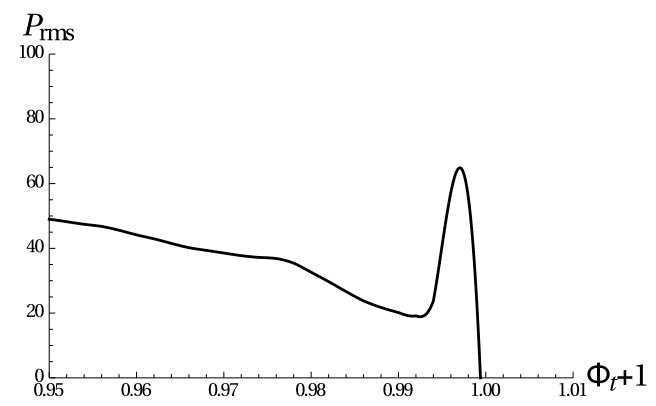

(a) $D_{a}=0$.

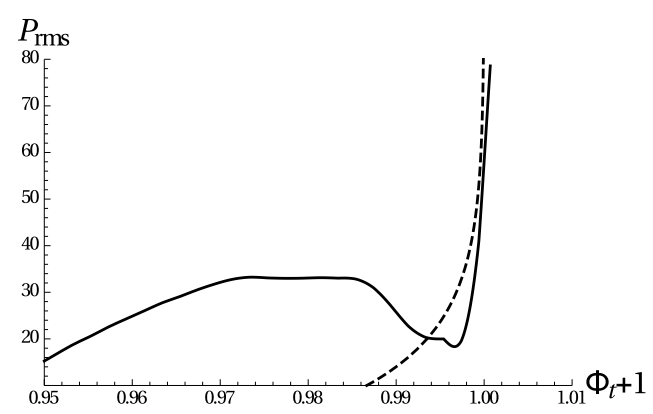

(c) $D_{c}=1.0$.

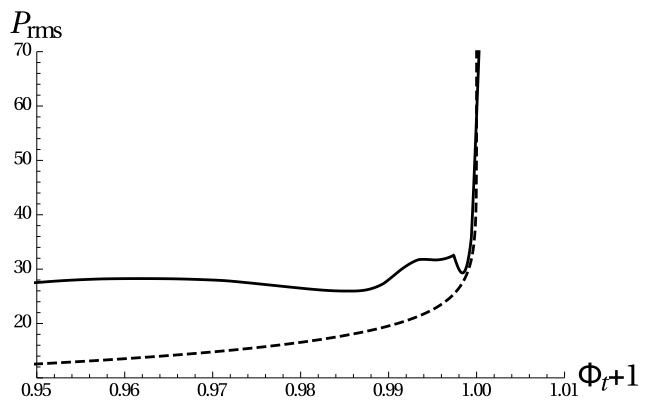

(b) $D_{b}=0.3$.

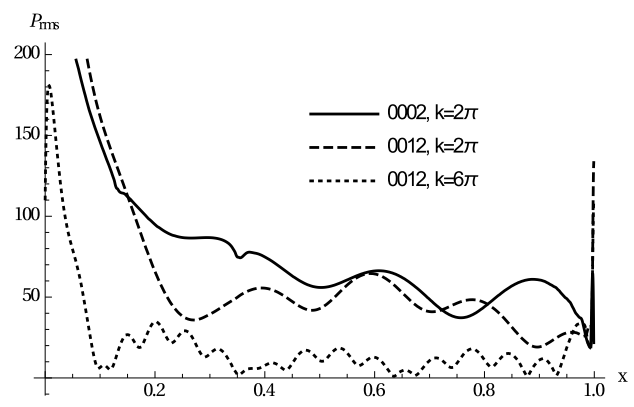

(d) Numerical surface pressure along the full chord.

Figure 3: a)-c) singularities in the pressure jump across the trailing edge of the aerofoil as $\Phi_{t} \rightarrow 0_{-}$. Solid lines are the numerical results, dashed lines model $\Delta p=D\left(-\Phi_{t}\right)^{-1 / 2}$. d) full chord-length numerical surface pressure. 
agreement, and we have not had to alter the unsteady Kutta condition imposed on the analytical solution. For case b), relaxing the unsteady Kutta condition alters the SPL predominantly in the upstream region, $\theta \in(\pi / 2, \pi)$, and overall the resulting analytical curve matches the numerical result more closely (the peaks and troughs are more aligned, and the magnitudes are more accurate). Neglecting the unsteady Kutta condition in case c) has a similar effect to case b) in the upstream region by increasing the overall SPL. This also gives better agreement with the numerical result, however a further increase of the SPL in the upstream region would be required for a good agreement. This is perhaps due to the estimation of the pressure singularity in case c) being less accurate than in case b), so that we might expect neglecting the unsteady Kutta condition in case b) to yield a solution that is in better agreement with the numerical result than case c). We can see in (23) that increasing thickness or frequency increases the magnitude of the required extra term, implying that the singularity becomes more pronounced for thicker aerofoils at higher frequencies, and the unsteady Kutta condition may become increasingly important in these cases. We have also neglected the possibility that the pressure jump is singular downstream of the aerofoil as $\Phi_{t} \rightarrow 0_{+}$because this is inconsistent with the original analytical model.

We now discuss the accuracy of the analytical solution; it is obtained by using the method of matched asymptotic expansions therefore the solution is the first two terms, $O(1)$ and $O(\epsilon \sqrt{k})$, is an asymptotic series. Hence the analytical solution has errors of $O\left(k^{-1}, \epsilon\right)$ in the amplitude and phase. In Figure 4, the analytical solution when neglecting the unsteady Kutta condition over-predicts the noise directly upstream of the aerofoil $(\theta=\pi)$ when compared to both the analytical solution with the unsteady Kutta condition and the numerical result. In the analytical solution, the upstream continuous pressure condition is imposed for the leading-edge solution at $O(1)$ and $O(\epsilon \sqrt{k})$. The trailing-edge solution is then found at $O\left(k^{-1 / 2}\right)$, and creates a discontinuity of pressure upstream (much like the leading-edge solution created a discontinuity of pressure downstream). To correct this, one would have to find further corrections to the leading-edge solution of $O\left(k^{-1}, \epsilon\right)$ which are negligible in the current analysis. We therefore expect the analytical solution (both with and without the unsteady Kutta condition) to have a discontinuity of pressure directly upstream which is not correct, and that this discontinuity is different when the unsteady Kutta condition is included or neglected. The analytical solution also neglects the $O\left(\epsilon^{2}\right)$ effects of the perturbation of leading-edge stagnation point position (this could also 


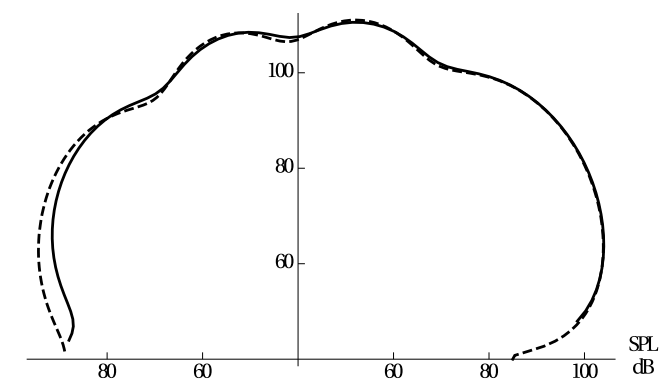

(a) Case a) NACA 0002, $k=2 \pi$.

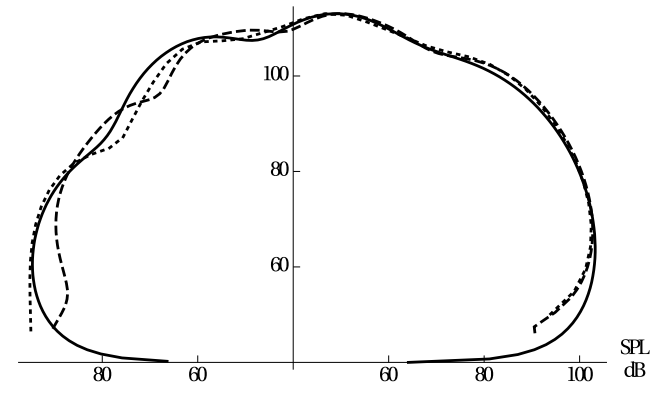

(b) Case b) NACA 0012, $k=2 \pi$.

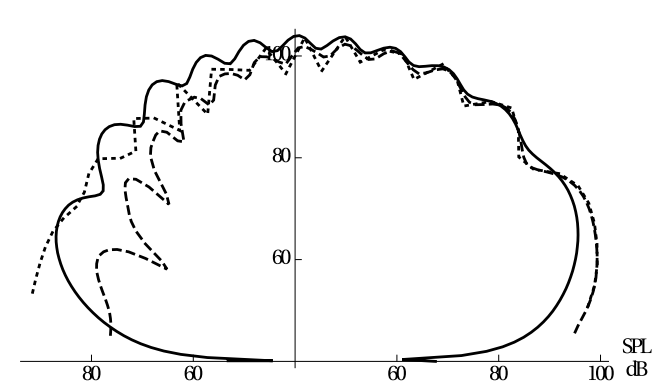

(c) Case c) NACA 0012, $k=6 \pi$.

Figure 4: Comparison of SPL at a radial distance of 100 chord lengths from the aerofoil leading edge. Solid line is the numerical result, dashed line is the analytical solution with the unsteady Kutta condition imposed, and dotted line is the analytical solution without the unsteady Kutta condition but with a singularity in the pressure at the trailing edge fitted to that seen in the numerical result.

be corrected with smaller asymptotic terms). For these two reasons, we anticipate a small region, of angular extent $O\left(\epsilon^{2} k\right)$, close to the leading edge, $\theta=\pi$, where the asymptotic solution is not accurate; this is approximately 0.09 radians for case b) and 0.27 radians for case $c$ ), which is consistent with the regions of over-predicted noise in Figure 4 when the unsteady Kutta condition is neglected.

\subsection{Sensitivity of the Upstream Acoustics to the Singularity}

In this subsection we investigate the sensitivity of the far-field upstream acoustic directivity to the level of singularity in the pressure jump at the trailing edge. As seen in the previous subsection, a singularity of the form $\left(-\Phi_{t}\right)^{-n}$, for $1 / 2<n<1$ yields significant differences in the far-field upstream directivity pattern. We now consider various other singularities with 


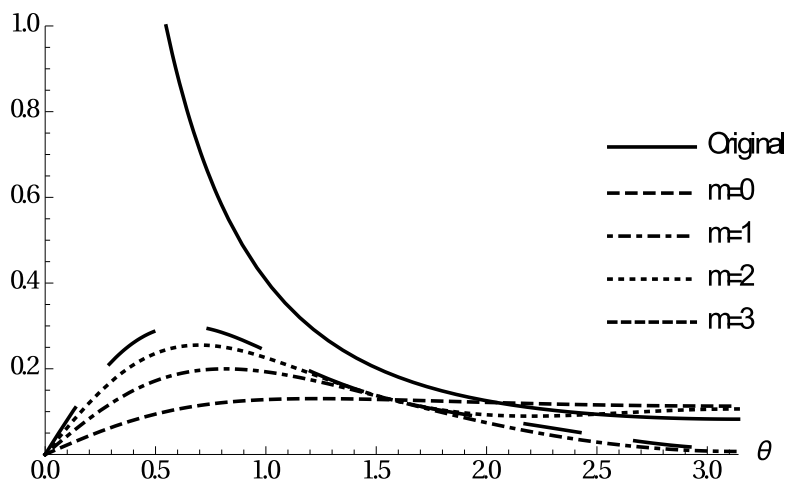

Figure 5: Magnitude of the original trailing-edge solution with the unsteady Kutta condition imposed (solid line) and the additional trailing-edge terms included if $E \neq 0$ as functions of polar angle $\theta$ measured from the downstream direction. The legend denotes the degree of the polynomial, $E$, where all coefficients have been set to one. In all cases, $M_{\infty}=0.6$.

$n>1$ by allowing $E(\lambda)$ to be a polynomial of degree $m$, for various $m \geq 1$, where $m$ is the integer strictly greater than $n-1 / 2$. Since the coefficients of the polynomial would be obtained by matching the singularity to numerical results which are not available, for illustration here we set all coefficients to one.

Figure 5 illustrates why only the upstream far-field acoustics are modified when we introduce a constant function $E(m=0)$. For any non-zero $E(\lambda)$ the values of the additional terms are much smaller than the original trailingedge solution for small values of $\theta$ (in the downstream region near the wake) whereas in the upstream region the contributions from the additional $E(\lambda)$ terms are similar in size to the original solution. For $m=0$ (the case we discussed in the previous subsection) we see that the new terms only become comparable to the original terms for $\theta \gtrsim \pi / 2$, therefore it is only in this region that we see a noticeable difference to the far-field directivity. The greater the singularity (larger values of $m$ ), the greater the additional terms are in the downstream region and the wider the region of $\theta$ values over which the additional terms are of comparable size to the original solution. We therefore expect that for stronger singularities, the effect on the far-field directivity would be seen in a wider region, beginning at a smaller value of $\theta$ than $\pi / 2$. Results in a portion of the downstream region would be unaffected by the singularity. The original trailing-edge field rescatters the leading-edge 
field, and acts to ensure continuous pressure across the wake, therefore is significant at $\theta=0$. The additional trailing-edge field is generated by the scattering of the (unphysical) pressure singularity by the trailing-edge and hence has a directivity proportional to $\sin (\theta / 2)$, which radiates upstream and therefore will not significantly affect results in the downstream direction.

\section{Conclusions}

We have compared an asymptotic analytical solution for the sound generated by gust-aerofoil interaction with a numerical model. The numerical model does not impose the unsteady Kutta condition, whilst the analytic solution has been found both with and without the unsteady Kutta condition imposed. The analytical solution that gives the best comparison to the numerical result is one in which the unsteady Kutta condition is not imposed at the trailing edge, allowing for an unsteady pressure jump singularity across the aerofoil as we approach the trailing edge. This singularity, which arose in the numerical results, has been implemented into the analytical solution by neglecting the unsteady Kutta condition that is applied during the analysis of the inner trailing-edge region, which is solved using the Wiener-Hopf method. The (analytical) effect of neglecting the unsteady Kutta condition is an additional acoustic term radiating from the trailing edge that ought not to be there. This term augments the total far-field noise in such a way as to give better agreement with the numerical results, indicating that numerical far-field results include an unphysical scattering of a pressure singularity at the trailing edge if a singularity in pressure is permitted at the trailing edge.

We have investigated the sensitivity of the far-field acoustic results to the level of singularity that could be present at the trailing edge in a numerical solution, and found that different regions of the far-field acoustic directivity can be affected by different singularities, governed by where the additional term from the Wiener-Hopf analysis is comparable to the original (unsteady Kutta condition imposed) solution. The downstream region saw little effect of including or excluding the unsteady Kutta condition, but the sound in the upstream region was modified significantly since the additional term takes a $\sin (\theta / 2)$ directivity pattern.

We can therefore conclude that neglecting the unsteady Kutta condition for gust-aerofoil interaction in steady uniform flow when aerofoils have non-zero thickness does have a significant impact on the predicted levels of far-field noise, and this effect seemingly increases with gust frequency and 
aerofoil thickness. It is therefore vital that numerical models correctly address the pressure at the trailing edge of aerofoils when considering unsteady interaction noise if they wish to accurately predict the far-field acoustics especially in the upstream observer direction. Alternatively, due to difficulties in applying an unsteady Kutta condition numerically, the result in this paper could be used to correct numerical far-field acoustic predictions where an unsteady Kutta condition has not been imposed, by subtracting the relevant far-field contribution generated by the scattering of the numerical pressure singularity at the trailing edge.

\section{Acknowledgements}

This work was supported by a Research Fellowship at Sidney Sussex College, Cambridge, UK (LA). Preliminary results from this paper were presented as part of AIAA Paper 2015-3265 at the 21st AIAA/CEAS Aeroacoustics Conference.

[1] Allampalli, V., Hixon, R., Nallasamy, M. \& SaWyer, S. D. 2009 High-accuracy large-step explicit Runge-Kutta (HALERK) schemes for computational aeroacoustics. Journal of Computational Physics 228, 3837-3850.

[2] Amiet, R. K. 1975 Acoustic radiation from an airfoil in a turbulent stream. Journal of Sound and Vibration 41, 407-420.

[3] Amiet, R. M. 1989 Gust response for flat-plate airfoils and the Kutta condition. AIAA Journal 28, 1718-1727.

[4] Ayton, L. J. 2014 Asymptotic Approximations for the Sound Generated by Aerofoils in Unsteady Subsonic Flows. PhD thesis, University of Cambridge.

[5] Ayton, L. J. \& PeAke, N. 2013 On high-frequency noise scattering by aerofoils in flow. Journal of Fluid Mechanics 734, 144-182.

[6] Basu, B. C. \& Hancock, G. J. 1978 The unsteady motion of a twodimensional aerofoil in incompresible inviscid flow. Journal of Fluid Mechanics 87, 159-178.

[7] Bender, C. M. \& Orszag, S. A. 1978 Advanced Mathematical Methods for Scientists and Engineers. Springer. 
[8] Bose, N. 1994 Explicit Kutta condition for an unsteady twodimensional constant potential panel method. AIAA Journal 32, 10781080 .

[9] Crighton, D. G. 1985 The Kutta condition in unsteady flow. Annual Review of Fluid Mechanics 17, 411-445.

[10] Davi, D., Marretta, R. M. A. \& Milazzo, A. 1997 Explicit Kutta condition for unsteady two-dimensional high-order potential boundary element method. AIAA Journal 35, 1080-1081.

[11] Devenport, W. J., Staubs, J. K. \& Glegg, S. A. L. 2010 Sound radiation from real airfoils in turbulence. Journal of Sound and Vibration 329, 3470-3483.

[12] Geyer, T., Sarradj, E. \& Giesler, J. 2012 Application of a beamforming technique to the measurement of airfoil leading edge noise. Advances in Acoustics and Vibration 2012, 1-16.

[13] Gill, J., Zhang, X., Joseph, P. \& Node-Langlois, T. 2013 Effects of real airfoil geometry on leading edge gust interaction noise. 19th AIAA/CEAS Aeroacoustics Conference, Berlin, DE, .

[14] Goldstein, M. E. 1978 Unsteady vortical and entropic distortions of potential flows round arbitrary obstacles. Journal of Fluid Mechanics 89, 433-468.

[15] Grace, S. M. 2001 Unsteady blade response: the BVI model vs. the gust model. 7th AIAA/CEAS Aeroacoustics Conference, Maastricht .

[16] Hixon, R. 2000 Prefactored small-stencil compact schemes. Journal of Computational Physics 165, 522-541.

[17] Hixon, R., Golubev, V., Mankbadi, R. R., Scott, J. R., SAWYeR, S. \& NAllasamy, M. 2006 Application of a nonlinear computational aeroacoustics code to the gust-airfoil problem. AIAA Journal 44, 323-328.

[18] Howe, M. S. 1978 A review of the theory of trailing edge noise. Journal of Sound and Vibration 61, 437-465. 
[19] Howe, M. S. 2003 Theory of Vortex Sound. Cambridge University Press.

[20] Hu, F. Q., Hussaini, M. Y. \& Manthey, J. L. 1996 Low-dissipation and low-dispersion Runge-Kutta schemes for computational acoustics. Journal of Computational Physics 124, 177-191.

[21] Liu, P., Bose, N. \& Colbourne, B 2002 A Broyden numerical Kutta condition for an unsteady panel method. International Shipbuilding Progress 49, 263-273.

[22] Lockard, D. P. \& Morris, P. J. 1998 Radiated noise from airfoils in realistic mean flows. AIAA Journal 36, 907-914.

[23] Myers, M. R. 1987 Effect of airfoil mean loading on high-frequency gust interaction noise. PhD thesis, University of Arizona.

[24] Myers, M. R. \& Kerschen, E. J. 1995 Influence of incidence angle on sound generation by airfoils interacting with high-frequency gusts. Journal of Fluid Mechanics 292, 271-304.

[25] Myers, M. R. \& Kerschen, E. J. 1997 Influence of camber on sound generation by airfoils interacting with high-frequency gusts. Journal of Fluid Mechanics 353, 221-259.

[26] Noble, B. 1998 Methods Based on the Wiener-Hopf Technique for the Solution of Partial Differential Equations. Chelsea Publications.

[27] Peake, N. \& Parry, A. B. 2012 Modern challenges facing turbomachinery aeroacoustics. Annual Review of Fluid Mechanics 44, 227-248.

[28] Sandberg, R. D. \& Sandham, N. D. 2006 Noise due to unsteady flow past trailing edges. European Conference on Computational Fluid Dynamics .

[29] Sandberg, R. D., Sandham, N. D. \& Joseph, P. F. 2007 Direct numerical simulations of trailing-edge noise generated by boundary-layer instabilities. Journal of Sound and Vibration 304, 677-690.

[30] Scott, J. R. 2004 Benchmark solutions for computational aeroacoustics (CAA) code validation. NASA Technical Report 2004, NASA/TM2004-213386. 
[31] Thwaites, B. 1960 Incompressible aerodynamics: an account of the theory and observation of the steady flow of incompressible fluid past aerofoils, wings, and other bodies. Dover Publications.

[32] Tsai, C-T. 1992 Effect of Airfoil Thickness on High-Frequency Gust Interaction Noise. PhD thesis, University of Arizona. 